\title{
Análisis
}

\section{La apertura financiera y la participación extranjera en el sistema bancario mexicano, 1994-2005}

DOI: 10.32870/mycp.v8i26.262

\section{Introducción}

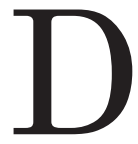
esde los años sesenta las políticas de liberalización financiera, y en particular la apertura de la cuenta de capitales de la balanza de pagos, son el componente más polémico de los programas de reformas estructurales. Se esperaban sustanciales beneficios económicos que incluían una mejor asignación de recursos, una mayor diversificación del riesgo de los inversionistas y la eliminación de las restricciones financieras de las economías nacionales. Sin embargo, las primeras experiencias — Corea del Sur a finales de los años ochenta, Argentina y Chile en la década siguiente- y los programas de ajuste estructural de "segunda generación" aplicados en las economías en desarrollo desde mediados de los años ochenta fueron rápidamente seguidos de "crisis gemelas" en la balanza de pagos y el sistema bancario. ${ }^{2}$

* Investigadora del Departamento de Estudios del Pacífico, Cucsh-Universidad de Guadalajara y miembro del SNI.
Geneviève Marchini*

México no pudo escapar del patrón de liberalización financiera-crisis gemelas que indica la literatura. Bajo el marco de un programa de estabilización y ajuste estructural patrocinado por el FMI (1988-1992), las autoridades lanzaron reformas financieras aceleradas que comprendieron la esfera financiera interna (1988-1992) y la apertura de la cuenta de capitales (1989-1992). Las medidas centrales para el sistema bancario incluyeron la liberalización de las tasas de interés, la eliminación del crédito selectivo, el regreso a un esquema de bancos múltiples y de grupos financieros, y, finalmente, la privatización de la banca (1991-1992). Al mismo tiempo, la apertura de la cuenta de capitales permitió la inversión extranjera en los mercados de bonos públicos internos y en el mercado accionario (1989-1990), autorizó a las empresas mexicanas las emisiones de títulos en los mercados internacionales (1989) y facilitó la inversión extranjera directa (IED) - a través del nuevo Reglamento de Inversión Extranjera de 1989 y de la nueva ley de inversión extranjera de 1993.

Si bien se liberalizó mucho su funcionamiento, el sistema bancario no fue incluido 
entre los primeros sectores abiertos a la IED. En 1994 los lineamientos del Tratado de Libre Comercio de América del Norte (TLCAN) sólo permitían la participación minoritaria de la inversión extranjera en el sistema. Después del fracaso de los inversionistas locales en establecer un sistema bancario que cumpliera con las reglas mínimas de eficiencia y estabilidad - evidenciado con la crisis bancaria de 1994-1995-, las autoridades cambiaron a fondo su postura porque permitieron la ampliación —en dos etapas-de la participación extranjera en el capital del sistema bancario (1995 y 1998).

Esta modificación de la política mexicana hacia la IED en el sector bancario correspondía plenamente a las nuevas recomendaciones emitidas por los organismos financieros internacionales. La apertura del sistema bancario a la IED ha sido promovida por estas instituciones después de las costosas crisis bancarias que sufrieron numerosas economías emergentes. Se esperaba una serie de beneficios para el sistema bancario y para la economía en general: en el corto plazo el primer objetivo era capitalizar mejor al sector que fue debilitado por la crisis; en el mediano plazo se esperaba que la mayor participación extranjera elevara la eficiencia de las instituciones a través de la introducción de nuevas tecnologías y mejoras en la gestión así como la creación de un mayor nivel de competencia en el sistema; y finalmente, en un plazo más amplio, se esperaba que la IED pudiera fortalecer la calidad de la asignación de recursos realizada por el sistema bancario y volver más estable el acceso al financiamiento internacional, lo cual permitiría a su vez alcanzar un mayor nivel de inversión y crecimiento económico.

Este artículo presenta los resultados de la apertura de la banca mexicana a la inversión extranjera. La primera sección analiza el cronograma de la apertura del sistema bancario y la importancia que ha adquirido la participación extranjera en el capital de la banca. La segunda sección estudia la evolución del crédito otorgado al sector privado: el aspecto más polémico del desempeño de la banca extranjera en nuestro país. La tercera sección muestra los alcances de la participación extranjera a nivel macroeconómico y reflexiona sobre el balance general de estas instituciones en México.

\section{La apertura y penetración de las instituciones extranjeras en el sistema bancario mexicano}

El sistema bancario fue abierto tardíamente a la participación de inversionistas extranjeros. En efecto, las reformas liberalizadoras que se iniciaron en 1989 no autorizaron la apertura del sistema bancario a la IED y, a diferencia de lo que ocurrió en otras industrias como la de telecomunicaciones, el proceso de 1991-1992 —a través del cual el gobierno regresó los bancos al sector privado- se realizó sin la participación de extranjeros. Los motivos de esta decisión han sido analizados en términos de la economía política de las reformas: la voluntad manifestada por las autoridades de maximizar los ingresos monetarios provenientes de la privatización bancaria les llevó a ofrecer a los posibles compradores locales un sistema poco competitivo que garantizara una alta rentabilidad y permitiera rembolsar con prontitud las deudas contraídas para adquirir los bancos. ${ }^{3}$ Por lo mismo, la apertura del sistema a la participación extranjera contemplada en el TLCAN estaba limitada por estrictas barreras: en 1994 la participación de la IED se limitaba a $30 \%$ del capital de un banco individual 
y sólo podía tener control en instituciones cuya participación del mercado no excedía $1.5 \%$ de los activos totales del sistema. Colectivamente, la participación de los bancos extranjeros no podía superar $8 \%$, una proporción que se podía elevar a $15 \%$ al final de un periodo de transición de seis años (2000), después del cual el Estado mexicano se reservaría el derecho de congelar las nuevas adquisiciones por tres años si la participación extranjera superaba $25 \%$ del mercado (Haber, 2004).

El desplome bancario posterior a la crisis de 1994-1995 en la balanza de pagos, propició un cambio radical en la estrategia de las autoridades mexicanas hacia la IED en el sistema bancario. De hecho, las necesidades de capitalización de los bancos y la insuficiencia de capitales mexicanos deseosos de invertir en este sector hicieron que el gobierno mexicano abriera el capital del sistema en dos etapas: en febrero de 1995 —en medio de la crisis- las autoridades autorizaron la participación mayoritaria de la inversión extranjera en el capital de todos los bancos menos en los tres más grandes; y en diciembre de 1998 esta medida se extendió a los tres bancos mencionados con lo cual se abrió la puerta a la participación extranjera dominante.

Este cronograma de apertura define tres etapas en la penetración de la banca extranjera en el país: ${ }^{4}$ en la primera etapa de 1994-1995 se establecen oficinas de representación de bancos extranjeros o subsidiarias destinadas a atender a la clientela corporativa de mayoreo; en la segunda, que abarca desde mediados de 1995 hasta fines de 1998, la banca extranjera adquiere participaciones en bancos mexicanos pequeños y medianos que atienden al público en general, es decir, en la banca "de menudeo"; finalmente, la tercera etapa, que se inicia en 1999, corresponde a la entrada masiva del capital extranjero en los tres grandes bancos de menudeo.

En consecuencia, la participación de las instituciones extranjeras — sólo controlaba $1 \%$ del capital del sistema bancario mexicano en 1994- se elevó hasta alcanzar $19 \%$ en $1999,24 \%$ en 2000 y $90 \%$ a partir de 2001 (Calderón, 2002). Las operaciones más importantes tuvieron lugar a partir de 2000 bajo la forma de "fusiones y adquisiciones" transfronterizas. Destacaron la compra de 100\% de Serfin por el español Banco Santander Central Hispano (BSCH) en mayo de $2000 ;{ }^{5}$ la toma de control de Bancomer por el Banco Bilbao Vizcaya Argentaria (BBVA) en agosto del mismo año con una participación accionaria de $32.2 \%$ y que fue completada en abril de 2004 cuando alcanzó 97.8\% del capital del banco; la adquisición de 99.9\% de las acciones de Banamex por Citigroup en agosto de 2001; y la compra de 99.5\% del capital de Bital por el banco inglés Hong Kong and Shanghai Banking Corporation (HSBC) en marzo de 2003 (Schultz, 2006). En lo que concierne al crédito bancario, la participación de la banca foránea se elevó de $11 \%$ en 1997 a $54 \%$ en 2001 y $83 \%$ en 2004 (gráfico 1).

\section{El debate sobre los beneficios de la participación extranjera y el crédito bancario al sector privado, 1994-2005}

Después de seis años de participación extranjera mayoritaria en el servicio de banca de menudeo en México, el balance de su aporte a la modernización y funcionalidad del sistema bancario mexicano es polémico. En México el debate ha abordado poco dos problemas interesantes: la pérdida de control sobre el sistema de pagos y del traslado de funciones y decisiones financieras claves 


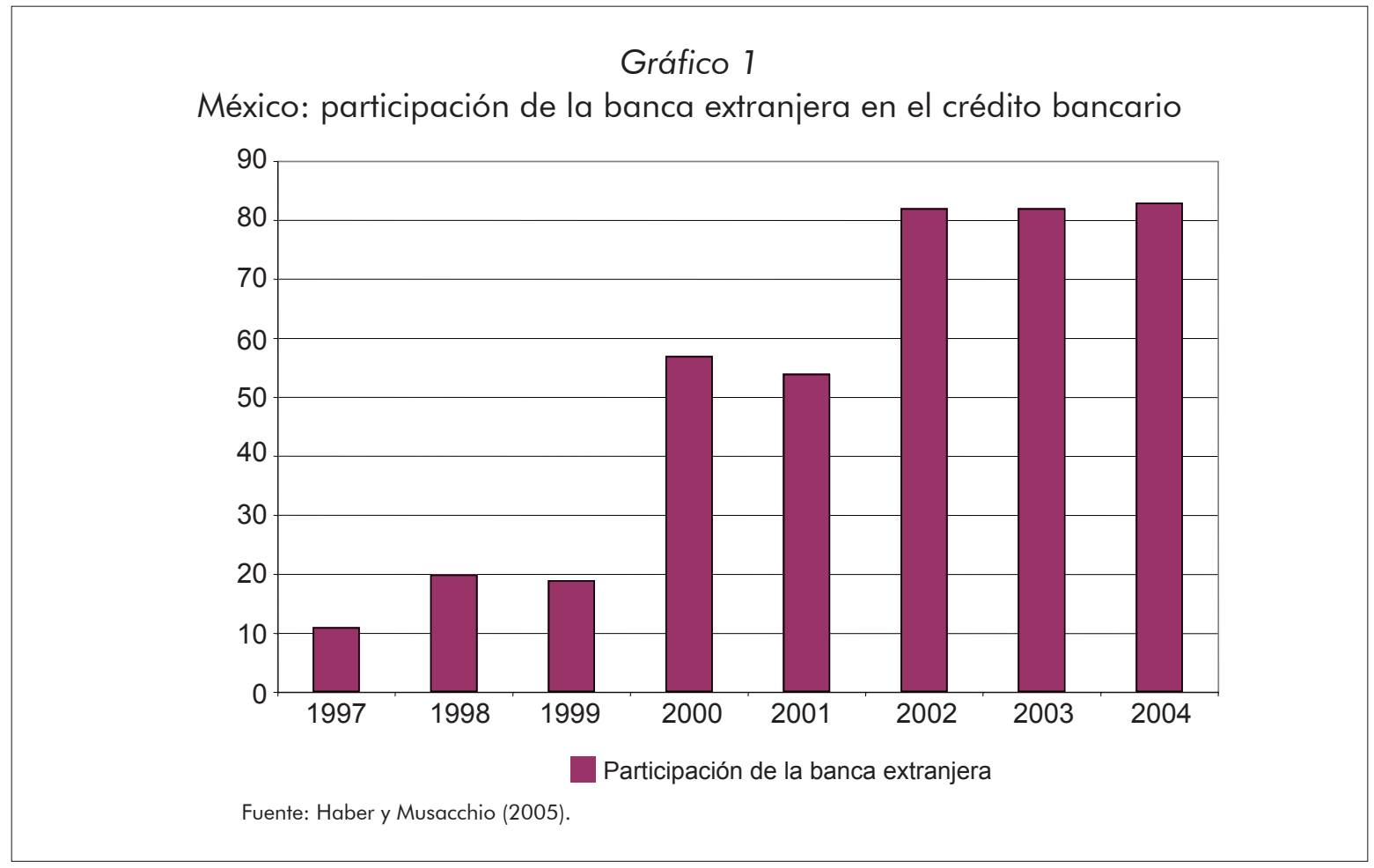

hacia centros internacionales, debido a una participación tan elevada de la inversión extranjera (Girón y Levy, 2005); y la protección de los depósitos locales en caso de una crisis del banco matriz. Estos temas han sido estudiados con detenimiento en las pocas economías desarrolladas que han autorizado un alto grado de propiedad extranjera de su sistema bancario. ${ }^{6}$

Más bien, el debate se ha centrado en la falta de beneficios visibles de esta elevada presencia extranjera; así, numerosos observadores han señalado que los costos de los servicios bancarios se han elevado sensiblemente, en particular las múltiples comisiones cobradas a los usuarios, y han subrayado aún más la prolongada contracción del crédito bancario al sector privado que experimenta el país desde 1995. Medido en términos reales, el crédito bancario al sector privado fue de $34 \%$ al final de 2004 contra 50\% en 1997 (Haber, 2004: 27). A su vez, el crédito bancario al sector público ya había superado $35 \%$ de los activos bancarios en $2001 .^{7}$

Esta contracción se puede observar en las gráficas 2 y 3 , las cuales presentan respectivamente el financiamiento real recibido por las familias y empresas entre 1994 y 2005 —en el primer año se estancó el crédito bancario-. De 1995 a 2005 la banca comercial ha contraído continuamente el crédito a las familias, que en 1994 representó $78.6 \%$ del financiamiento recibido por las familias y que, a precios constantes de 2002, fue de 673,385 millones de pesos (MDP). Además, en el periodo considerado la estructura del financiamiento de las familias se ha modificado profundamente, pues las fuentes alternativas de financiamiento — que incluyen a las Sociedades Financieras de Objeto Limitado y las entidades no-financieras como las tiendas departamentales - han elevado su oferta después de la desaparición del crédito bancario. A partir de 2003 la banca 
comercial volvió a elevar su oferta de crédito a las familias, primero bajo la forma de crédito al consumo - en particular, las tarjetas de crédito- y luego como crédito hipotecario.

Sin embargo, en 2005 el monto real de crédito bancario a las familias alcanzó un valor de 392,583 MDP a precios de 2002, es decir, alrededor de $72 \%$ del crédito que la banca mantenía con este sector en 1994; a su vez, este monto sólo constituía 41.6\% del total de financiamiento a las familias en 2005. Esta secuencia nos muestra que el proceso de contracción del crédito bancario ya tenía varios años cuando los principales bancos fueron adquiridos por las instituciones extranjeras, pero señala también que bajo su nueva conducción siguieron aplicando políticas de contracción del crédito a las familias y sólo introdujeron algunos cambios recientes.

Aunque el crédito a las familias parezca haber evolucionado de manera positiva en los últimos años, la situación del sector empresarial sigue siendo muy preocupante (gráfico 3). En este sentido, la contracción del crédito bancario iniciada en 1994-1995 sigue hasta la fecha (2005): el crédito a empresas o personas con actividad empresarial, que en 1994 fue de 1'600,937 MDP constantes de 2002, cayó a 389,111 MDP en 2005, es decir, a menos de la cuarta parte de su valor inicial. El reciente crecimiento del crédito ofrecido por la banca comercial a las familias no se ha producido en el caso de las empresas: en consecuencia, el crédito empresarial ha perdido importancia relativa en el total del crédito que la banca dirige al sector privado, ya que disminuyó de $75 \%$ del total en 1994 a menos de la mitad en 2005.

Las otras fuentes de financiamiento del sector empresarial han elevado su contribución, pero sólo han sustituido una parte del crédito de la banca comercial: el financiamiento total a las empresas ha

Gráfico 2

México: el financiamiento a las familias, 1994-2005

(En millones de pesos constantes de 2002)

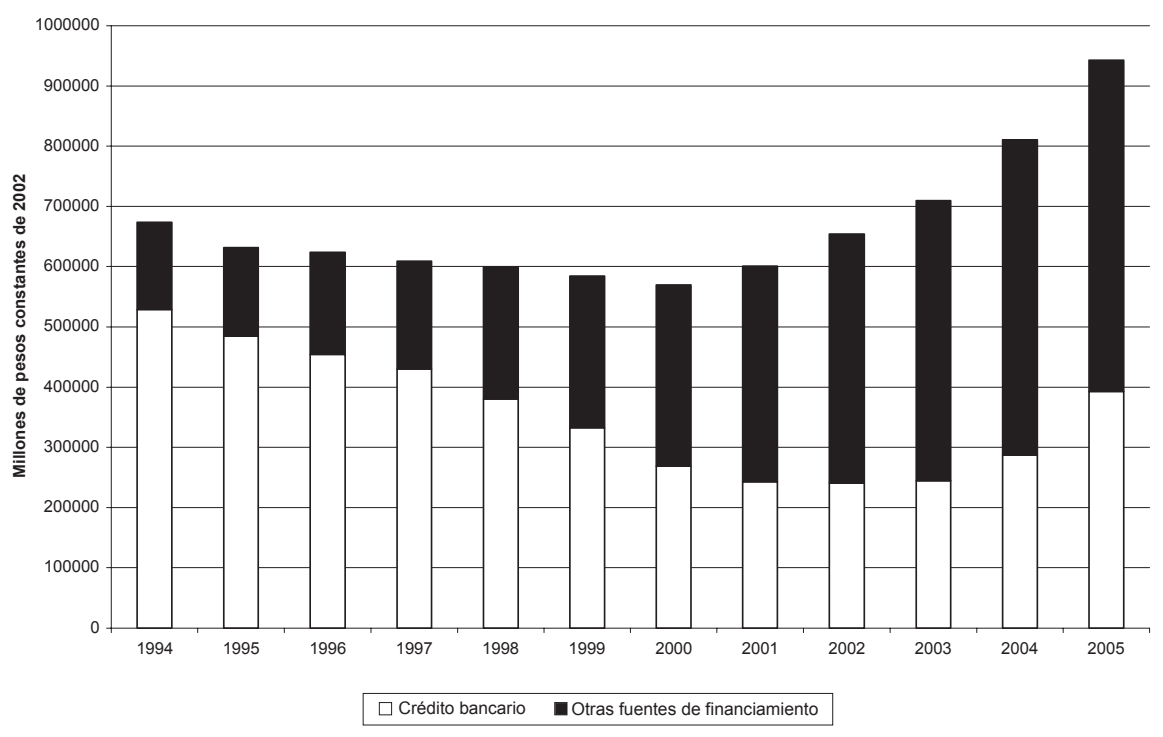

Fuente: elaboración de la autora con datos del Banco de México. 


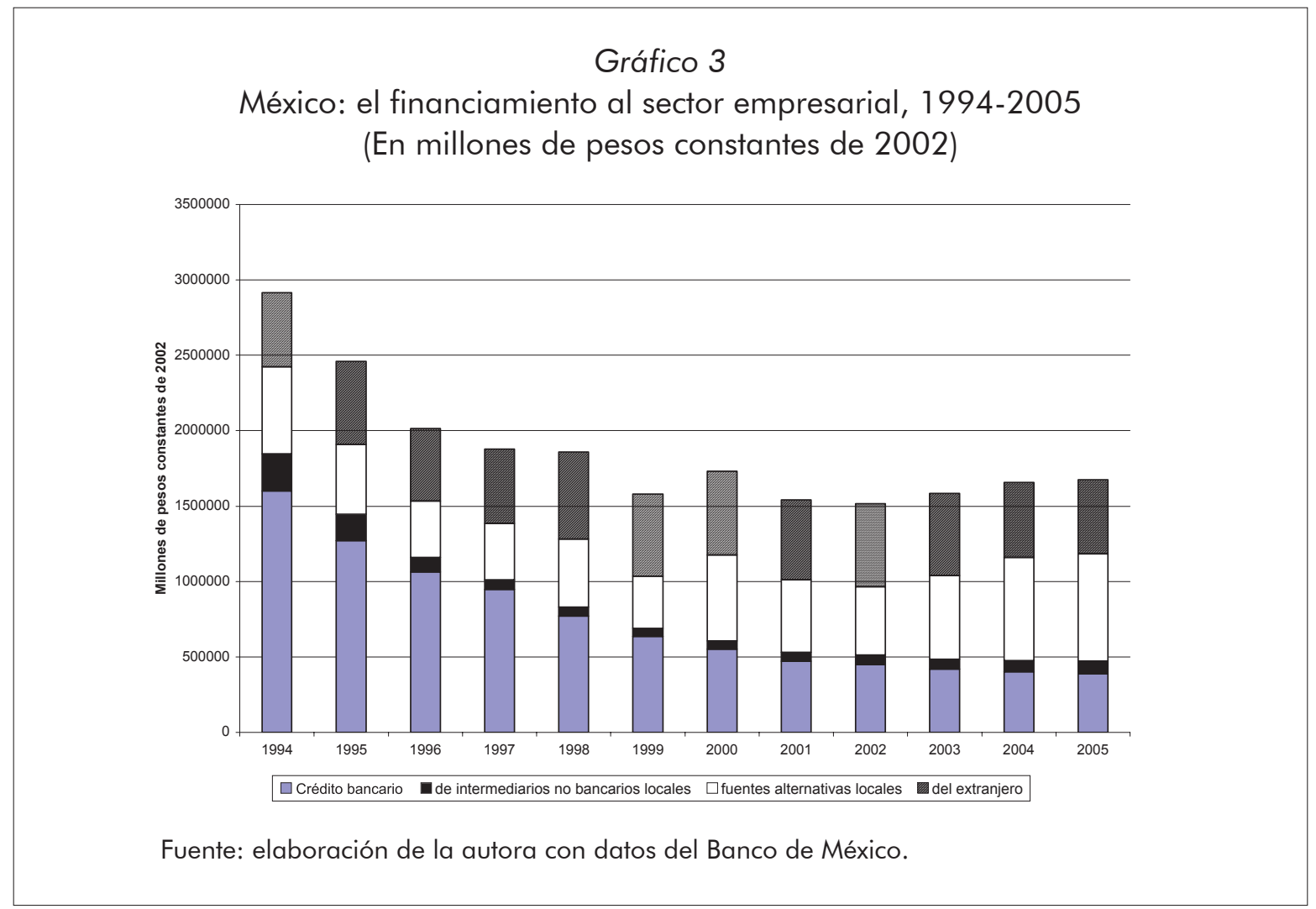

experimentado una severa caída seguida de un estancamiento. En diciembre de 2002 el sector recibió financiamiento real equivalente a $52 \%$ del de 1994 y el modesto repunte posterior situó esta proporción en $57 \%$ a finales de 2005 . Las fuentes alternativas que han permitido aminorar el impacto de la caída del crédito bancario al sector fueron, en primer lugar, el financiamiento externo, que ha crecido más que proporcionalmente desde los primeros años que siguieron a la crisis, y luego, las fuentes alternativas locales, entre los cuales destaca -en los últimos años- el incipiente mercado interno de bonos corporativos. Inversamente, los demás intermediarios no-bancarios locales contrajeron su contribución real, al igual que la banca.

La banca atiende cada vez menos a las empresas mexicanas y cuando lo hace tien- de a concentrarse en un puñado de grandes corporaciones: en efecto, la proporción que representan los trescientos acreditados mayores en la cartera comercial de la banca se ha elevado en los últimos años en todos los bancos más importantes del sistema. Ahora esa proporción es mayoritaria en todos estas instituciones, especialmente en los dos principales: 93.8\% para BBVA Bancomer y $97.6 \%$ para Banamex, así como para Scotiabank Inverlat (100\% en 2005); sólo en el caso del banco Santander Serfin esta proporción es inferior a $60 \%$ de la cartera comercial. Cabe señalar que el único banco con capital mexicano mayoritario ha seguido una política similar, pues los acreditados mayores, que representaron $30.6 \%$ de su cartera comercial en 2002, constituyeron casi $75 \%$ de ésta en 2005 (tabla 1 ). En suma, el crédito cada vez más escaso de la banca comercial para las empresas 
no-financieras del país se ha dirigido con creces hacia las grandes empresas.

\section{Tabla 1}

México: el porcentaje de acreditados mayores / cartera comercial

\begin{tabular}{lrrrr}
\hline & \multicolumn{2}{c}{2002} & \multicolumn{2}{c}{2005} \\
\cline { 2 - 5 } & 50 & 300 & 50 & 300 \\
& mayores & mayores & mayores & mayores \\
\hline BBVA Bancomer & 62.0 & 87.9 & 67.9 & 93.8 \\
Banamex & 55.1 & 79.1 & 67 & 97.6 \\
Serfin $^{\mathrm{a}}$ & 33.7 & 36.8 & 37.5 & 57.3 \\
Santander & 76.9 & 85.0 & $\mathrm{ND}$ & $\mathrm{ND}$ \\
HSBC Bital $^{\mathrm{b}}$ & 31.5 & 57.6 & 57.4 & 73.9 \\
Mercantil del $_{\text {Norte }}$ & & & & \\
Scotiabank & 22.3 & 30.6 & 49.2 & 74.4 \\
Inverlat & & & & \\
\hline
\end{tabular}

a. Santander Serfin a partir de 2005.

b. Bital en 2002 y HSBC a partir de 2005.

Fuente: elaboración de la autora con datos del Banco de México.

Las encuestas periódicas realizadas por el Banco de México confirman esta tendencia: desde que este instrumento existe, la proporción de empresas que afirman no recurrir al crédito bancario ha aumentado constantemente. Cuatro años después de la crisis, a finales de 1998, 61.7\% de las empresas encuestadas declaró que no recibió crédito bancario, una proporción que subió a $75.7 \%$ en 2005 , más de diez años después. Simultáneamente, las empresas declararon recurrir cada vez menos al financiamiento de la banca pública de desarrollo, que en 2005 atendió a sólo 1.7\% de los encuestados (contra 3.8\% en 1998) y cada vez más al crédito de los proveedores $-60.2 \%$ de los encuestados en 2005 y $47.3 \%$ en 1998 (tabla 2).

En suma, la participación de la IED en el sistema bancario mexicano no ha permitido levantar la restricción que la notoria insuficiencia del financiamiento interno hace pesar sobre las empresas mexicanas y, por ende, sobre el crecimiento económico del país. A pesar de la presencia mayoritaria de bancos extranjeros, el sistema bancario mexicano le sirve de poco al crecimiento del PIB. Así mismo, su tamaño es aún más reducido que el promedio latinoamericano, una región caracterizada — con contadas excepciones- por su subdesarrollo financiero. Algunos trabajos recientes se han enfocado a analizar este problema y han procurado medir el impacto de la entrada de la banca internacional al país. La sección siguiente presenta algunos de los hallazgos de dichos trabajos.

\section{Tabla 2}

México: los resultados de la encuesta trimestral del mercado crediticio

(Cifras al cuarto trimestre de cada año)

\begin{tabular}{lrrr}
\hline & 1998 & 2002 & 2005 \\
\hline Fuentes de financiamiento: & 100 & 100 & 100 \\
Proveedores & 47.3 & 55.9 & 60.2 \\
Banca comercial & 24.6 & 20.3 & 17.2 \\
Banca internacional & 6.8 & 5 & 2.7 \\
Grupo corporativo & 12.1 & 12.1 & 13.0 \\
Bancos públicos de desarrollo & 3.8 & 1.9 & 1.7 \\
Matriz & 3.6 & 3.7 & 3.8 \\
Otras & 1.8 & 1.1 & 1.4 \\
Empresas que: & & & \\
reciben crédito bancario & 38.3 & 30.9 & 24.3 \\
no utilizan el crédito bancario & 61.7 & 69.1 & 75.7 \\
Empresas que proveen & & & \\
financiamiento: & 76.9 & 77.3 & 77.9 \\
a clientes & 77.2 & 76.2 & 75 \\
a proveedores & 11 & 10.3 & 12.4 \\
a empresas del grupo & 11 & 13.5 & 12.6 \\
\hline
\end{tabular}

Fuente: Banco de México.

\section{El impacto de la penetración de la banca extranjera en México}

El impacto de la participación mayoritaria extranjera en la banca mexicana se puede abordar en dos niveles: en primer lugar, 
a nivel microeconómico, de las instituciones, se ha buscado medir sus efectos a través de las variables de productividad, capitalización, calidad de la cartera de crédito (Schultz, 2006), eficiencia administrativa (disminución de costos), precio del crédito y márgenes de intermediación así como desempeño financiero (Schultz, 2006). Algunos estudios comparativos indican que la participación extranjera tiende a elevar las cuatro primeras variables y a disminuir las últimas tres. Por otro lado, en términos macroeconómicos resulta interesante dilucidar si la presencia de la banca extranjera ha modificado la oferta de crédito bancario, si el comportamiento de estos bancos difiere significativamente del de los bancos que permanecen bajo control local, y los factores que explican las diferencias y similitudes en la conducta de las instituciones.

A nivel microeconómico se destaca que el impacto de la mayor participación extranjera en México ha sido positivo pero limitado. En primera instancia, la principal aportación de la banca extranjera consistió precisamente en capitalizar las instituciones, lo cual constituía el primer objetivo de las autoridades mexicanas; su segunda contribución fue disminuir la proporción de créditos de mala calidad del sistema; ambos cambios permitieron dar más estabilidad al sistema (Schultz, 2006: 6 y 33). Ahora bien, en términos de productividad y de eficiencia del sistema bancario, las evidencias indican un impacto bastante limitado de la penetración extranjera. Los bancos, en general, se han vuelto más rentables a través de un incremento de sus ingresos por el alza de las comisiones cobradas y del margen de intermediación, y la reducción de costos vinculados al aprovisionamiento de los créditos de mala calidad. Sin embargo, la participación extranjera ha afectado muy poco a diversos indicadores de productividad, sin ningún efecto comprobable sobre los costos administrativos o el personal empleado (Schultz, 2006: 5).

Por otro lado, el modelo aplicado por Haber y Musacchio tampoco encontró diferencias significativas entre bancos extranjeros y locales en términos de costos o de rentabilidad, si bien los autores avanzan que los bancos extranjeros aplicaron un proceso de "screening" o análisis crediticio más intenso de sus acreditados potenciales, lo cual se reflejó en una menor tasa de créditos de mala calidad (Haber y Musacchio, 2005:4).

La falta de competencia en el seno del sistema bancario mexicano, donde predominan algunas instituciones, es una de las hipótesis más atractivas que se manejan para explicar el pobre resultado de la entrada de instituciones extranjeras: en su mercado doméstico la presión de la competencia obliga a estas instituciones a disminuir sus costos y elevar sus niveles de productividad, pero en México no hacen lo mismo porque la competencia es débil (Schultz, 2006).

A nivel macroeconómico varios estudios no encuentran impacto alguno de la 
penetración extranjera sobre la oferta de crédito al sector privado y las políticas crediticias de las instituciones adquiridas. Las políticas de asignación de recursos de la banca extranjera no difieren de las aplicadas por los bancos que quedaron en manos del capital mexicano. Además, se concluye que existen factores que afectan a los bancos en general y que impactan decisivamente sobre su oferta de crédito al sector privado al volver a estas instituciones "adversas al riesgo". Entre las causas mencionadas, cabe hacer especial mención de los problemas relacionados con la calidad del marco legal y del sistema de impartición de justicia, que debilitaron la capacidad de los bancos para hacer valer sus derechos en caso de incumplimiento del acreditado, lo cual los obligó a reducir su oferta de crédito al sector privado; por otro lado, los incentivos creados por el rescate bancario debilitaron el estímulo para prestar a este sector, debido a la presencia de pagarés públicos muy rentables y de cero riesgo en los activos de los mayores bancos.

\section{Conclusión}

Este texto revisó el proceso de apertura del sistema bancario mexicano a la inversión extranjera directa que tomó lugar después de la crisis bancaria de 1994-1995. Hemos visto que la penetración de la banca extranjera en México presenta características únicas, por la rapidez con la cual se hizo y el alcance que tiene actualmente - más de $80 \%$ del capital bancario- en todos los bancos de importancia sistémica menos uno. El análisis de los resultados de este proceso concluye que las instituciones extranjeras contribuyeron sobretodo a la capitalización y la estabilización del sistema, pero que han tenido poco impacto sobre la eficiencia de las instituciones y, más aún, no han ayudado a resolver las severas restricciones de financiamiento externo que sufren las empresas mexicanas, en especial las pequeñas y medianas. En este sentido, la aportación específica de la banca extranjera ha sido irrelevante hasta la fecha: han seguido el patrón establecido por los bancos nacionales antes de la entrada de la IED: la disminución del crédito al sector privado, en especial a empresas nofinancieras, y el rápido aumento del crédito al sector público. En suma, la entrada del capital extranjero en el sistema bancario no ha sido hasta ahora el remedio a los problemas de subdesarrollo financiero que México padece. Para el país es incalculable el costo de la prolongada contracción de la oferta de crédito - la cual perdura hasta la fecha para el sector empresarial-y hasta cierto punto la falta de acción de parte de las autoridades es incomprensible. my

\section{Notas}

1 Estos programas dieron lecciones importantes del fracaso de las experiencias tempranas y dedicaron mayor atención a la secuencia y al ritmo de las reformas, y ubicaron - por lo general- la apertura de la cuenta de capitales al final de las medidas para minimizar el riesgo y los costos para la economía.

2 Siguen en debate los efectos a mediano y largo plazos de la apertura de la cuenta de capitales sobre el crecimiento y el bienestar económicos. Entre las contribuciones relevantes véanse Tornell, Westerman y Martínez (2003) y Bekaert, Harvey y Lundblad (2005). Por otro lado, la posición del FMI en relación con estas políticas ha sido examinada recientemente por su oficina de evaluación independiente (Independent Evaluation Office) cuyos resultados se puede consultar en FMI (2005).

3 Esta elevada rentabilidad está asociada a las rentas provenientes de la estructura oligopólica del sistema. Muchos bancos fueron comprados a crédito, con frecuencia aportado a los accionistas-compradores por las mismas instituciones 
que se adquirieron. Véase Marchini (s/a) para un análisis más exhaustivo de este proceso.

4 Véase la periodización de Haber y Musacchio (2005), la cual difiere de la que se presenta en este trabajo.

5 En marzo de 2003 Bank of America adquirió una participación de $24.9 \%$ en el capital de este banco.

6 Considere por ejemplo la actitud proteccionista de la participación nacional en el capital del sistema bancario que prevalece en la Unión Europea, y la postura que han debido adoptar las autoridades neo-zelandesas en materia de supervisión y reglamentación para proteger el sistema bancario local en el caso de que ocurriera una crisis bancaria en Australia, país donde se origina la propiedad de cuatro bancos de importancia sistémica para el sector bancario neo-zelandés. Véase FMI (2004).

7 En 1998 los pagarés públicos constituían gran parte del llamado "crédito al sector público" $-25 \%$ de los activos bancarios-, los cuales respaldaban la transferencia de créditos al Fobaproa. A finales de 2004 esta proporción había bajado a menos de $10 \%$ de los activos bancarios, y el resto estaba constituido por inversiones en títulos de deuda pública interna (15\% de los activos bancarios) y préstamos al sector público. Véase FMI (2005).

\section{Referencias bibliográficas}

Bekaert, Gert, C. Harvey y C. Lundblad (2005), "Does Financial Liberalization Spur Growth?",
Journal of Financial Economics, núm. 77, pp. 3-55.

Calderón, A. (2002), "Los bancos españoles y América Latina: ¿̇se necesitan mutuamente?", documento preparado para la conferencia Spanish Investment in Latin America.

FMI (2004), "New Zealand: Financial System Stability Assessment", Country Report, núm. 04/126, Washington, DC.

(2005), "Report on the Evaluation of the IMF's Approach to Capital Account Liberalization", disponible en http://www.img.org.

_ (2005), Mexico: Selected Issues 2005, Washington, DC.

Girón, A. y N. Levy (2005), México. Los bancos que perdimos, UNAM, México, DF.

Haber, S. (2004), "Mexico's Experiment with Bank Privatization and Liberalization, 1991-2003", mimeo, Department of Economics, Stanford University, disponible en http://www.stanford.edu/ haber/.

Haber, S. y A. Musacchio (2005), "Foreign Banks and the Mexican Economy, 1997-2004", mimeo, Department of Economics, Stanford University, disponible en http://www.stanford.edu/ haber/.

Marchini, G. (2003), "Financial Reforms in Mexico: Looking for the Causes of Failure", Journal of APEC Studies [Corea del Sur], vol. 5, núm. 1, pp. 55-84.

Schultz, H. (2006), "Foreign Banks in Mexico: New Conquistadores or Agents of Change?", mimeo, University of Pennsylvania, disponible en http://fic. wharton.upenn. edu/fic/papers/06/0611.pdf.

Tornell, A., F. Westerman y L. Martínez (2003), "Liberalization, Growth, and Financial Crises: Lessons from Mexico and the Developing World", Brookings Papers on Economic Activity, núm. 2, pp. 1-112. 